\title{
In Vitro Simulation of Tissue Back-Pressure for Pen Injectors and Auto-Injectors
}

\author{
Yildiz, Arda; Lenau, Torben Anker
}

Published in:

Journal of Pharmaceutical Sciences

Link to article, DOI:

10.1016/j.xphs.2019.03.035

Publication date:

2019

Document Version

Peer reviewed version

Link back to DTU Orbit

Citation (APA):

Yildiz, A., \& Lenau, T. A. (2019). In Vitro Simulation of Tissue Back-Pressure for Pen Injectors and AutoInjectors. Journal of Pharmaceutical Sciences, 108(8), 2685-2689. https://doi.org/10.1016/j.xphs.2019.03.035

\section{General rights}

Copyright and moral rights for the publications made accessible in the public portal are retained by the authors and/or other copyright owners and it is a condition of accessing publications that users recognise and abide by the legal requirements associated with these rights.

- Users may download and print one copy of any publication from the public portal for the purpose of private study or research.

- You may not further distribute the material or use it for any profit-making activity or commercial gain

- You may freely distribute the URL identifying the publication in the public portal

If you believe that this document breaches copyright please contact us providing details, and we will remove access to the work immediately and investigate your claim 


\section{In Vitro Simulation of Tissue Back-Pressure for Pen Injectors and Auto-Injectors}

Authors: MSc Arda Yildiz ${ }^{1}$ and Associate Professor Torben Anker Lenau ${ }^{2}$

Suggested running head: In vitro Simulation of Tissue Back-Pressure

${ }^{1}$ Department of Mechanical Engineering, Technical University of Denmark, Kgs. Lyngby, Denmark

2 Department of Mechanical Engineering, Technical University of Denmark, Kgs. Lyngby, Denmark 


\section{Abstract:}

Purpose: The aim of this project was to show that tissue back-pressure can be measured in vitro using a simple pneumatic model.

Methods: A thorough literature study revealed four relevant papers all describing in vivo studies. One of these studies where the subcutaneous tissue back-pressure was determined in 11 patients was used as a reference for the present work. A pneumatic model capable of simulating the back-pressure and the diffusion of drug during subcutaneous injection was developed. The in vitro model was tested using the same type of pen injector as used in the reference study.

Results: Comparison of the results revealed that the measured pressure in the in vitro experiments were similar to the subcutaneous tissue back-pressure measured in vivo. G30 $0.3 \times 8.0 \mathrm{~mm}$ and G32 0.23/0.25 x 4.0 mm needles were used for the in vitro experiments, whereas a G31 $0.25 \times 6.0 \mathrm{~mm}$ needle was used for the in vivo experiments. This is one possible explanation of approximately $30 \mu \mathrm{L} / \mathrm{s}$ higher flow rates for the in vitro experiments compared to the in vivo experiment.

Conclusion: The low-complexity model allows repeated measurements, and provides a stable data output paving the way for measuring subcutaneous back-pressure in vitro. 


\section{Introduction:}

Pen injectors and auto-injectors are common devices used for patients' self-administration of medicine. The route of drug administration is most commonly either subcutaneously (SC) or intra muscularly (IM). However, self-treatment with needles is often by patients considered a not pleasant experience. The components used within the device contribute to the treatment experience. While most pen injectors are powered by the user, are auto-injectors mainly spring-powered devices. The injectors need to overcome different parameters such as friction inside the primary container and the needle, needle size, and injection rate. Furthermore, the human tissue generates a certain pressure during injection, which has a major impact on the composition of the mechanical parts within the injectors. This pressure, referred to as subcutaneous back-pressure, can serve as an indicator for changes in subcutaneous mechanical properties that can lead to pain during injection. In order to mechanically design and make injectors as user-friendly and painless as possible, optimization of the injectors is necessary. The optimization of the injectors can adjust the dosing by the relationship of pharmacokinetics and pharmacodynamics. The relationship of pharmacokinetics and pharmacodynamics connects the concentration-time profile evaluated by pharmacokinetics to the intensity of observed response as measured by pharmacodynamics [1]. Unfortunately, testing devices in vivo are time consuming and expensive due to the required ethical approval. Therefore, it is attractive to have an in vitro model that can test pen injectors and auto-injectors against subcutaneous back-pressure fast in the laboratory without the need for ethical approval.

A literature search on the state-of-the-art knowledge on subcutaneous back-pressure was carried out. Five relevant articles were found together with a Ph.D. thesis [2-7]. The study by Allmendinger et al. calculated the tissue back-pressure by measuring the injection forces during subcutaneous injection [2]. This was done in vivo in 10 male Göttingen minipigs, since their subcutaneous layer resembles humans better than other investigated animals. Different Dextran solutions were used to achieve the desired viscosities. The used injection rates were constant at $0.025 \mathrm{~mL} / \mathrm{s}$, $0.1 \mathrm{~mL} / \mathrm{s}$, or $0.2 \mathrm{~mL} / \mathrm{s}$. The study concluded that the tissue back-pressure from the subcutaneous tissue contributing to injection forces measured during in vivo injections was determined to be between 0.1-12.9 $\mathrm{N}$ [2]. The study by Thomsen et al. was also made in vivo, but using 11 male human volunteers with Diabetes type 2 [3]. Injections were done in air and injection rates determined by recording the click sounds from the injector pen with a microphone. This was compared with injections in tissue, also determining the injection rates from the time between injector pen click 
sounds. FlexTouch $®$ pen injectors from Novo Nordisk A/S were used for injection. The pen injectors make a click-sound for each $10 \mu$ injected making it possible to calculate flow rate for the injections. Only 8 of the 11 patients in the reference in vivo study were used since data for three of the patients were omitted by the author due to unclear measurements $[3,4]$. The subcutaneous tissue back-pressure was measured to 190-998 mBar [4] and 150-900 mBar [3].

The study by Doughty et al. explains the ratio between injection speed and volume subcutaneously and concludes that the subcutaneous pressure decays to 0 several seconds after injection. Furthermore, they find that the subcutaneous pressure increases with increasing flow rate, but not with increasing dose volume [5]. The study by Mathaes et al. discusses current and publicly available data related to SC administration volumes, and conclude that 1.5 $\mathrm{mL}$ is commonly perceived as a limit for injection, but in reality higher volumes can be achieved by application of various techniques [6]. Völker et al. measured the subcutaneous pressure in the os sacrum area when people where laying down and found values 23-23.5 mm Hg = 30.7-31.3 mBar [7].

The present work uses the data from Thomsen et al. [3] as reference values for the subcutaneous tissue backpressure.

The following 4 research questions (RQ) were investigated: RQ1: Can the glass cartridge be reused for several experiments or does the friction increase?

RQ2: How much time does it take to make injection of a volume of $0.8 \mathrm{~mL}$ ?

RQ3: Which leakage rate is suitable for simulating drug diffusion into tissue?

RQ4: Are back-pressure and flow-rate measured in in vitro models similar to in vivo reference measurements?

\section{Materials and Methods:}

A pneumatic model was developed using a glass cartridge as injection site. The glass cartridge was pre-filled with liquid and sealed with a capsule having a rubber membrane through which needles were injected into the glass cartridge. The developed model is an airtight system with controlled leakage that mimics the diffusion of drug within the body. There is a $2.7 \mathrm{~mL}$ dead volume. The injections into the glass cartridge were made using Novo Nordisk FlexTouch $\AA$. The dimensions of the glass cartridge components were defined to be: 
Piston:

Diameter: $9.6 \mathrm{~mm}$

Height: $8.2 \mathrm{~mm}$

Cartridge:

ID: $9.3 \mathrm{~mm}$

OD: $11.0 \mathrm{~mm}$

Total height: $64.6 \mathrm{~mm}$

Height of cylindric part: $57.0 \mathrm{~mm}$

Cartridge with capsule:

Total height: $66.1 \mathrm{~mm}$ (depending on how tight it is closed).

Furthermore, the used glass cartridges are siliconised with baked-on silicone emulsion in order to make the piston move more easily by keeping the friction to a minimum. The developed model is presented in figure 1 . We used G30 $0.30 \times 8 \mathrm{~mm}$ and G32 $0.23 \times 4 \mathrm{~mm}$ needles for the injections. The reference study used a G31 $0.25 \times 6 \mathrm{~mm}$ needle, which were unavailable at the time of our study.

In total, four different experiments were made in order to answer the research questions. The aim was to have an endpressure on $900 \mathrm{mBar}$, because it was the maximum end-value from [3]. The injection time was recorded by clicksignals emitted from the pen injectors similar to the reference study.

The first experiment addresses RQ1 \& RQ2. In total 14 tests were done: 7 using the same glass cartridge and 7 using new glass cartridges every time. The second experiment addressing RQ3 measured the controlled leakage rate in the interval $1000 \mathrm{mBar}$ down to $50 \mathrm{mBar}$ for three different build in leakages. The rates were determined as the time in seconds for loss of pressure in the interval. The adjustable orifice at position 6 in figure 1 determines the leakage rates. The last two experiments address RQ4 replicating the in vivo data using the in vitro model. The G31 needles used in the reference study was not available and instead G30 needles were used in experiment 3 and G32 needles in experiment 4. The injection time in both experiments were recorded by the click-signals emitted from the pen injectors, as in the reference study. In experiment 3 patient 2, 4, and 7 were selected from the reference study because the same amount, $0.24 \mathrm{~mL}$, was injected into the patient during the in vivo study. Experiment 3 was repeated 7 times for low, medium and fast leakage. In experiment 4 patient 3, 5 and 7 were selected since they represent top, middle and bottom of the interval of flow rates. Experiment 4 was repeated 5 times. Statistical treatment was done using the JMP 
software [8]. Curves were fitted to the measured data points using a sigmoid logistic 3-parameter function with the following formula:

$$
y=\frac{c}{1+\operatorname{Exp}(-a(x-b))}
$$

where $a$ is the growth rate, $b$ the inflection point and $c$ the asymptote. Curves are compared statistically by looking at the means for the three parameters and assessing if they can be said to be different from an overall mean at a given confidence level.

\section{Results:}

$R Q 1:$ The results from the friction test are presented in figure 2 . The mean end-pressure was approximately $900 \mathrm{mBar}$ with a standard deviation of $25 \mathrm{mBar}$ in both cases, which justifies that the same glass cartridge can be used for all tests.

RQ2: For a $4.7 \mathrm{~mL}$ system volume the start pressure has to be $780 \mathrm{mBar}$ to achieve an end-pressure of $900 \mathrm{mBar}$ after injecting $0.8 \mathrm{~mL}$ liquid with a pen injector. The mean injection time for $0.8 \mathrm{~mL}$ was 4.41 seconds with a standard deviation of 0.60 seconds. The data is presented in figure 3.

$R Q 3:$ The results of the three different controlled leakage rates are presented in figure 4 . The leakage rate decreases for lower pressure.

RQ4: The results from experiment 3 are presented in figure 5. Different controlled leakage values (high, medium and low) were applied to the model in order to simulate the diffusion of injected liquid within the body. Due to the relative fast injection, the used leakage value is insignificant for the study, so medium leakage rate was applied in the following experiment. The best results were obtained for patient 2 and patient 4 from the reference study, where the percentage difference between the in vitro experiments and in vivo experiments were less than $1 \%$. The results were most diverse from the desired end-pressure for patient 7 from the reference study, which differed up to $3.8 \%$ between the in vitro experiments and in vivo experiments. This could be due to the relative low end-pressure, which can be more difficult for the model system to achieve. In general, the leakage rates performed approximately equal which was confirmed by statistically testing the curves for difference from the overall means. There was no evidence for difference at a $0.1 \%$ significance level as seen in table 1. 
The results from experiment 4 are presented in figure 6 and figure 7. Like the in vivo data the in vitro data is normalized in proportion to the lowest injected amount, which is $0.18 \mathrm{~mL}$, in order to have same conditions. Figure 6 shows the calculated flow rates from experiment 4 . The graphs have similar tendencies being relatively steady and flat for the first part of the injection, and then slightly more steep for the rest of the injection. The statistical analysis shows that the mean values for the curves cannot be said to be different from the overall mean with a significance level of $0.1 \%$ as seen in table 1.

Figure 7 compares the experiment 4 flow rates with the in vivo flow rates measured by Thomsen [3]. The mean endpressures measured in the model for patient $3 / 5 / 7$ was $956 / 986 / 397$ mBar compared with the in vivo measurements by Thomsen who measured it to $963 / 998 / 387 \mathrm{mBar}$. As seen, the smallest difference between in vitro and in vivo results was $7 \mathrm{mBar}$. When looking at the percentage difference, the results between the in vitro experiment and in vivo experiment only differs $0.73 \%$ for patient $3,1.20 \%$ for patient 5 , and $2.58 \%$ for patient 7 . In general the in vitro graphs seems to have a lager average flow rate but the statistical analysis of the mean values for the graph parameters (growth rate, inflection point and asymptote) can not see a difference at a significance level of $0.01 \%$.

\section{Discussion:}

As presented in figure 4 there are clear differences between the slow leakage rate, the medium leakage rate, and the fast leakage rate. There is approximately a factor five difference between the slow leakage rate and the fast leakage rate, a factor three difference between the slow leakage rate and the average leakage rate, whereas there is a factor two difference between the average leakage rate and the fast leakage rate. This emphasizes that higher pressure leads to higher leakage, which models that higher pressure leads to quicker diffusion of drug in the subcutaneous area. For the developed model, the medium leakage rate is used to simulate diffusion into tissue, since a design of experiment revealed that the diffusion rate was not a significant factor for this specific setup.

Even though the used leakage rate is insignificant in this study and medium leakage was used, the leakage has a practical and theoretical role. It is practical since the start-pressure is easier to achieve, and it has theoretical value 
because the diffusion of drug in the subcutaneous layer can vary from person to person. The slow leakage and fast leakage options can be used during the utilization of the device when testing different drug diffusion rates.

Comparing the in vivo and in vitro data it is seen that the values from the experiment with the G30 needle are higher compared to the results obtained by Thomsen. This can be explained with the different needle sizes used. Thomsen used a G31 needle, which is smaller than a G30 needle. Therefore, the flow rates for the in vitro experiment with the G30 needle are higher, since more liquid can pass the bigger needle.

As seen from figure 7 , the flow rates obtained from the in vitro experiments are higher than the flow rates obtained by the in vivo experiments. As mentioned, this could be due to different needle sizes used for the pen injectors. According to Hagen-Poiseuille equation, the length of the needle impacts the flow rate. The needle length in the in vitro experiment was $4 \mathrm{~mm}$ while the in vivo experiment needle was $6 \mathrm{~mm}$. Both shared the same inner diameter of 0.125 $\mathrm{mm}$. Additionally, it could also be due to the used injector. The timing of one click may not correlate directly to $10 \mu \mathrm{l}$ injected due to the break-loose point of FlexTouch ${ }^{\circledR}$ pen injectors. However, the one click $10 \mu$ l relationship is assumed in the reference study. Nevertheless, the red and blue curves follow the same tendency of falling flow rate in figure 7. 


\section{Conclusion:}

The present study shows that it is possible to simulate the subcutaneous tissue back-pressure in vitro using a pneumatic model. No previous work on in vitro modeling of tissue back-pressure was found. The developed model is based on airpressure in a closed system, where a glass cartridge is applied as injection site. Comparing the in vitro model and the reference in vivo results obtained by Thomsen et al. [3] revealed similar results. The difference in end-pressures found by comparing the results from the in vitro model with the in vivo experiments was only $0.14 \%-3.88 \%$. The flow rates were higher for the in vitro model, which could be linked to differences of needle dimensions, especially since higher flow rates were observed when a shorter needle was used. Further tests with different needles sizes are therefore required. However, a statistical analysis could not at a $0.1 \%$ significance level show that the in vitro and in vivo data were different giving a positive indication of a useful model. The developed model can be utilized to test the withstanding of pen injectors and auto-injectors during an injection and support development of the drive mechanism of injection devices. The experiments conducted are operating at low pressure. Therefore, small areas are necessary to simulate subcutaneous back-pressure. A little increase in the volume has a great impact on the system. If the dead volume was smaller than $2.7 \mathrm{~mL}$ as it is in the model, the model could also be used to test an injector's ability to withstand an excessive increasing pressure during injection. However, with the current design, this cannot be done. The model can be used to withstand an already generated back-pressure during injection.

\section{Acknowledgements:}

We are grateful for valuable supervision and sparing throughout the project from Principal Engineer Mads Schjøth Due and Principal Quality Engineer Carsten Schaufuss-Feddersen from NNE A/S. Additionally, thanks to Principal Consultant Benny Munk, Device Technology Engineer Bjarke Svendsen, Drug Delivery Compliance Engineer Michael Jensen, and Aseptic Design Engineer Zara Berthelsen, all from NNE A/S, for helping during this project. And thanks to Associate Professor Kaj-Åge Henneberg from The Technical University of Denmark for providing with a Greisinger manometer. Also a warm thank you to Teamleader Camilla Thyregod from DTU Compute for her kind help with the statistical analysis. The project was carried out as a graduate research project at DTU in collaboration with NNE A/S. 


\section{References:}

[1] Derendorf H, Lesko LJ, Chaikin P, et al. Pharmacokinetic/pharmacodynamics modeling in drug research and development. The Journal of Clinical Pharmacology, 40(12 Pt 2):1399-1418, 2000.

[2] Allmendinger A, Mueller R, Schwarb E, et al. Measuring tissue back- pressure - in vivo injection forces during subcutaneous injection. Pharmaceutical Research, 32(7):2229-2240, 2015.

[3] Thomsen M, Hernandez-Garcia A, Mathiesen J, et al. Model study of the pressure build-up during subcutaneous injection. Plos One, 9(8):e104054, 2014.

[4] Thomsen M. Subcutaneous Injections: Visualising and Optimising Device- Tissue Interactions. PhD thesis, 2015.

[5] Doughty DV, Clawson CZ, Lambert W, Subramony JA. Understanding subcutaneous tissue pressure for engineering injection devices for large-volume protein delivery. Journal of Pharmaceutical Sciences, 105(7):2105-2113, 2016.

[6] Mathaes R, Koulov A, Joerg S, Mahler HC. Subcutaneous injection volume of biopharmaceuticals-pushing the boundaries. Journal of Pharmaceutical Sciences, 105(8):2255-2259, 2016.

[7] Volker HU, Rolker N, Willy C. Interface pressure measurement in the treatment of pressure sores. Comparison with subcutaneous pressure values measured in healthy volunteers. Anaesthesist, 55(2):142-147, 2006.

[8] JMP Statistical Discovery from SAS, version 14.1.0, available from http:www.jmp.com, accessed 27. December 2018. 


\section{figures}

Table 1. Parameter estimates for curves fitted to data sets. Curves are fitted using a sigmoid logistic 3 parameter function and statistics are shown for an $\alpha$-level of 0.001 (0.1\% significance level).

\begin{tabular}{|c|c|c|c|c|c|c|c|c|c|c|}
\hline \multirow{2}{*}{$\begin{array}{l}\mathrm{Pa}- \\
\text { tient }\end{array}$} & & \multicolumn{3}{|c|}{ Growth rate } & \multicolumn{3}{|c|}{ Inflection point } & \multicolumn{3}{|c|}{ Asymptote } \\
\hline & & Lower limit & Estimate & Upper limit & $\begin{array}{c}\text { Lower } \\
\text { limit }\end{array}$ & Estimate & $\begin{array}{c}\text { Upper } \\
\text { limit }\end{array}$ & $\begin{array}{c}\text { Lower } \\
\text { limit }\end{array}$ & Estimate & $\begin{array}{l}\text { Upper } \\
\text { limit }\end{array}$ \\
\hline \multirow{2}{*}{3} & in vitro & $-3,36422$ & $-2,35302$ & $-0,53402$ & 0,951063 & 1,148211 & 1,344631 & 120,7075 & 151,9026 & 183,2088 \\
\hline & in vivo & $-4,27644$ & $-0,85664$ & 0,378204 & $-9,21177$ & 0,138154 & 11,50746 & $-741,865$ & 197,4473 & 1045,781 \\
\hline \multirow{2}{*}{5} & in vitro & $-4,6278$ & $-3,20858$ & $-1,47307$ & 1,154481 & 1,249636 & 1,36229 & 138,1802 & 153,4385 & 166,4527 \\
\hline & in vivo & $-7,63616$ & $-1,71385$ & 1,535284 & 0,569588 & 1,6429 & 1,947183 & 81,03129 & 123,7849 & 223,6016 \\
\hline \multirow{2}{*}{7} & in vitro & $-3,7763$ & $-1,33779$ & 1,480692 & $-1,31674$ & 0,804939 & 2,926617 & $-91,8896$ & 239,132 & 570,1536 \\
\hline & in vivo & $-5,37273$ & $-0,65697$ & 3,07712 & $-90808,1$ & $-13,3848$ & 90809,69 & $-6,1 e+10$ & 1020207 & $6,08 e+10$ \\
\hline
\end{tabular}

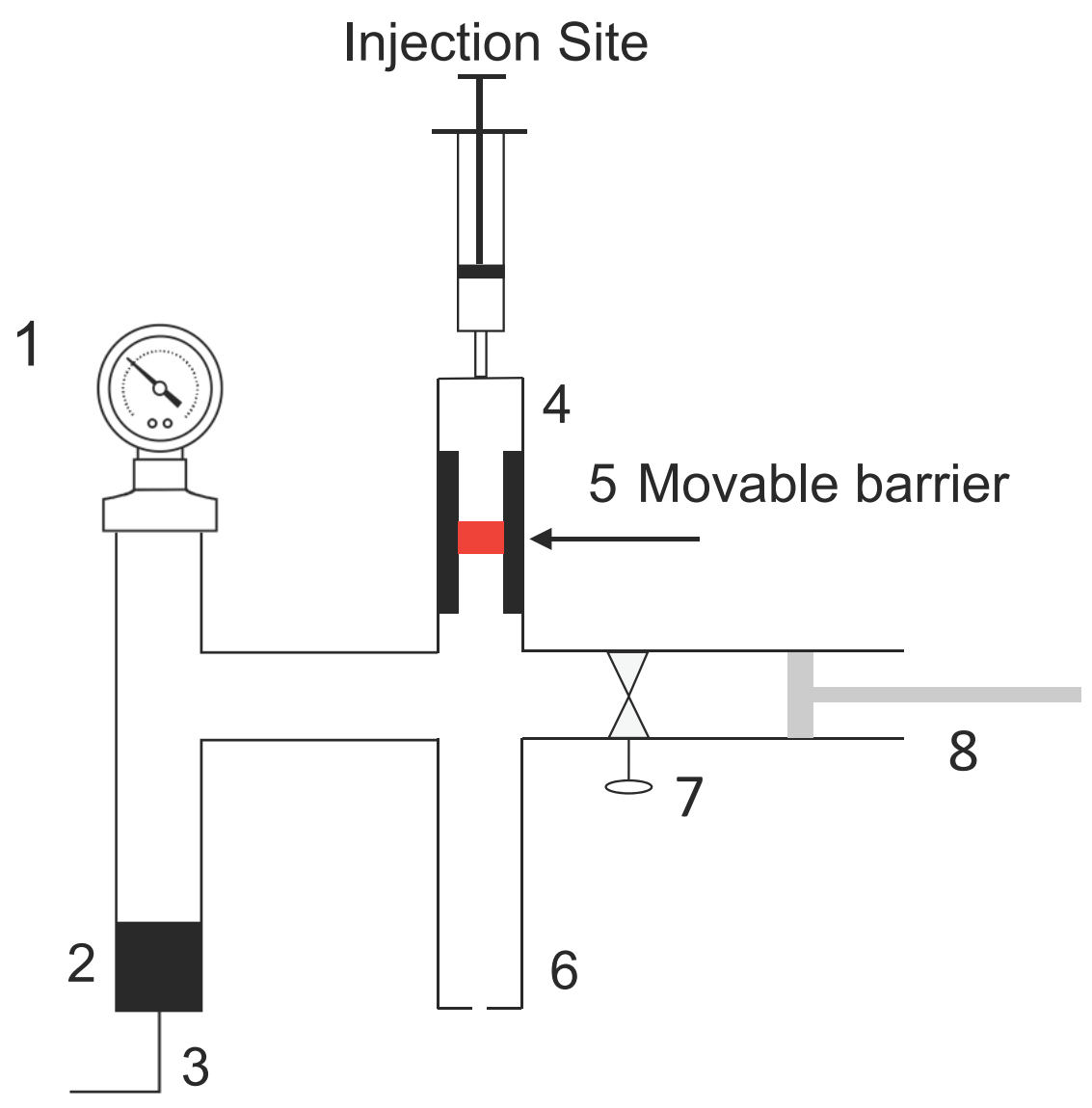

Figure 1: The sketch of the developed in vitro model: manometer for practicality (1), 0 to 2.5 bar pressure sensor (2), connection cable for pressure sensor (3), glass cartridge mounted in fixture as injection site (4), moveable barrier in glass cartridge (5), adjustable orifice for controlled leakage in the system (6), 1-piece ball valve to close the system after establishing pressure, tube fitting (7), and a syringe to establish initial start pressure (8). 


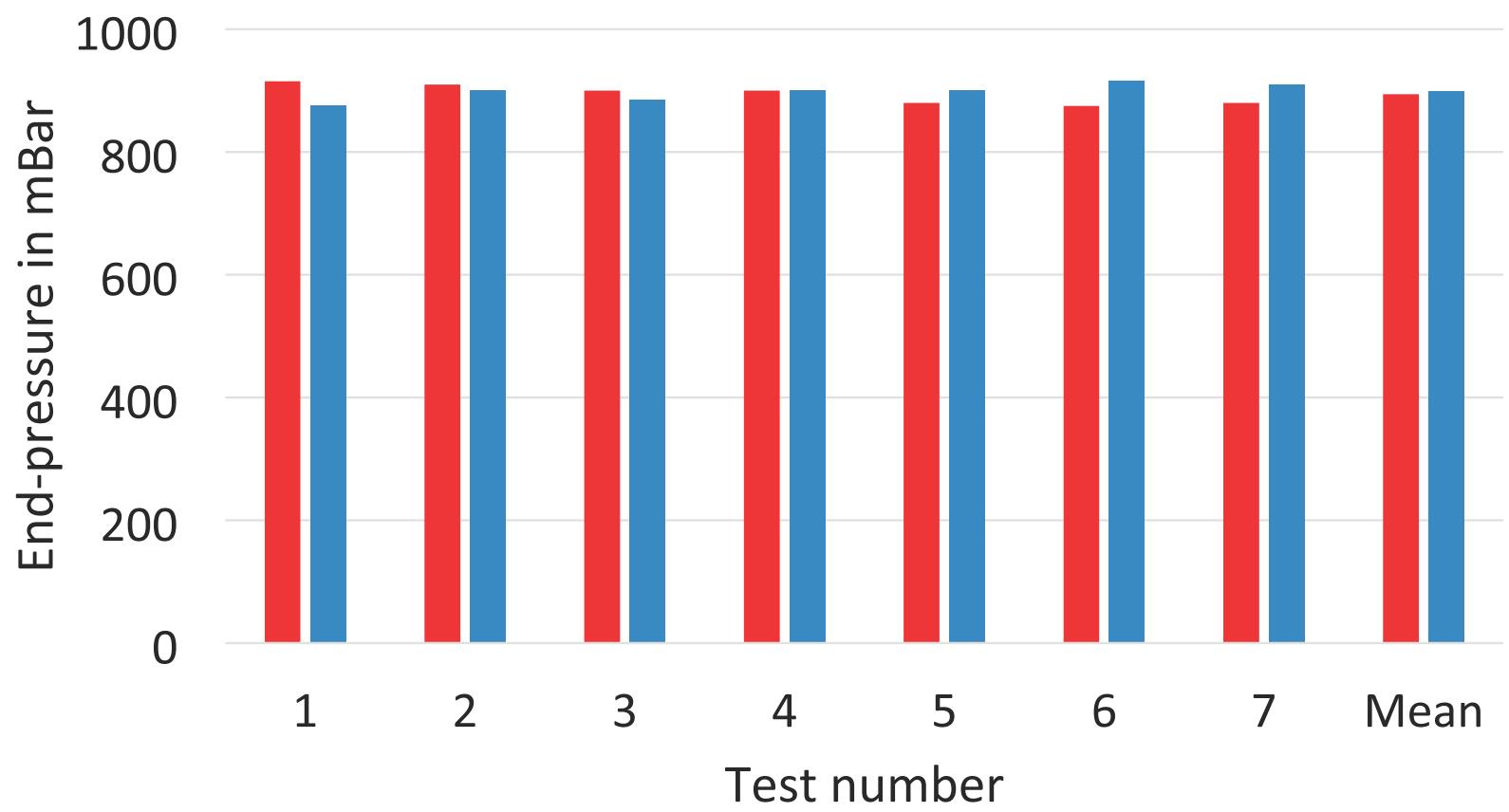

Figure 2: Friction test with same cartridge (red) compared to using different cartridges (blue). The standard deviation is $25 \mathrm{mBar}$ and the mean value when the same cartridge is used is $894 \mathrm{mBar}$, and for different cartridges is it $898 \mathrm{mBar}$.

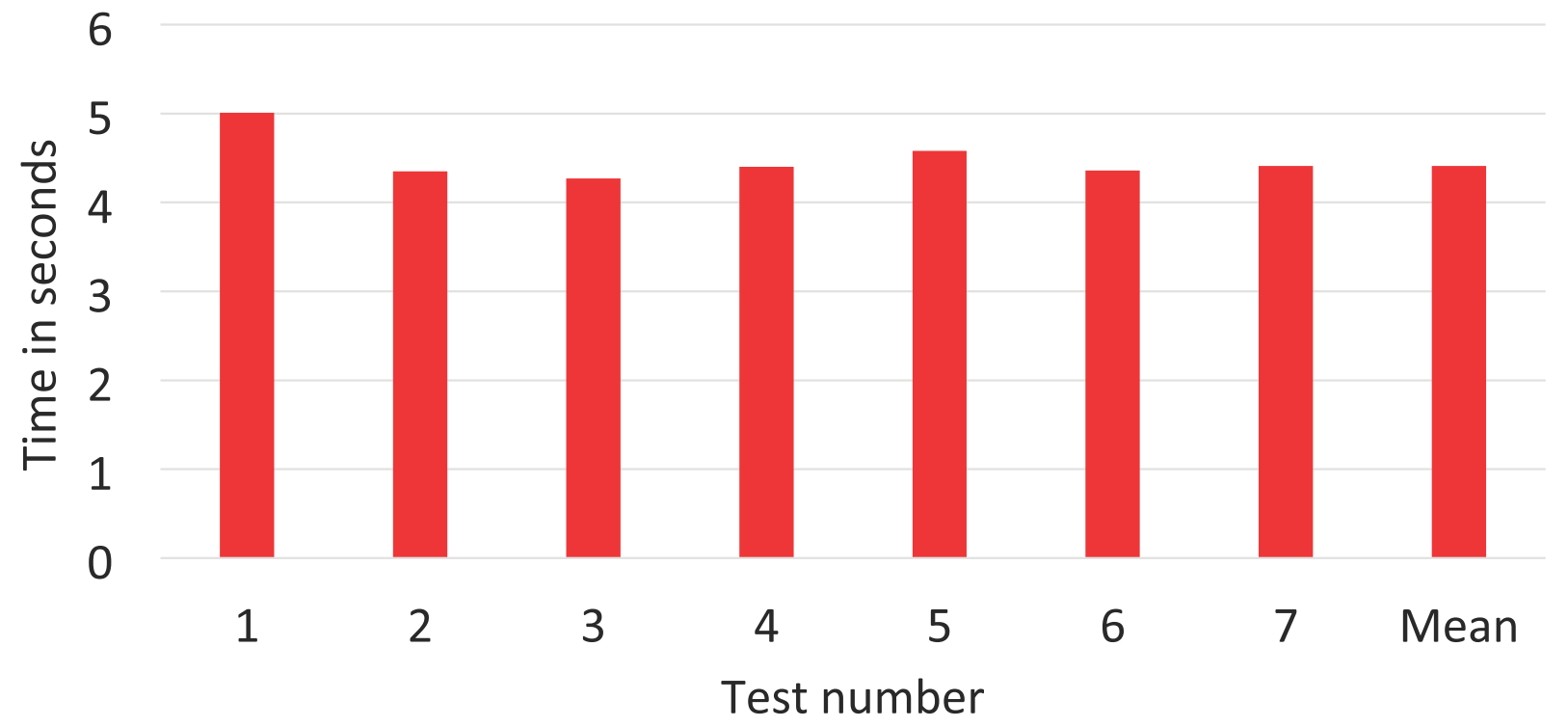

Figure 3: The injection time when injecting $0.8 \mathrm{~mL}$ using a pen injector and the same cartridge. The injection time varies between 4,2 seconds and 5.0 seconds. 


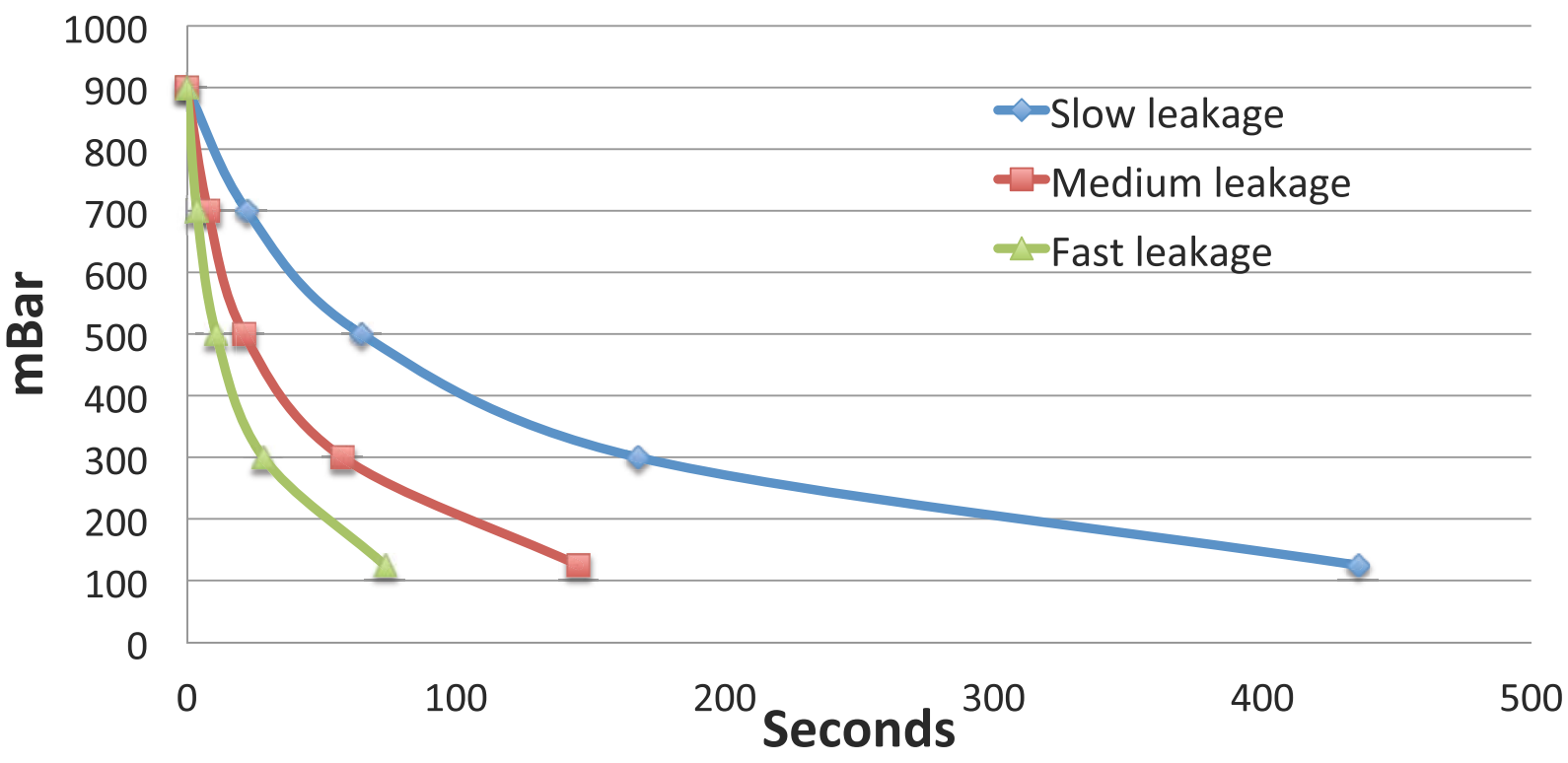

Figure 4: The three different leakage values.

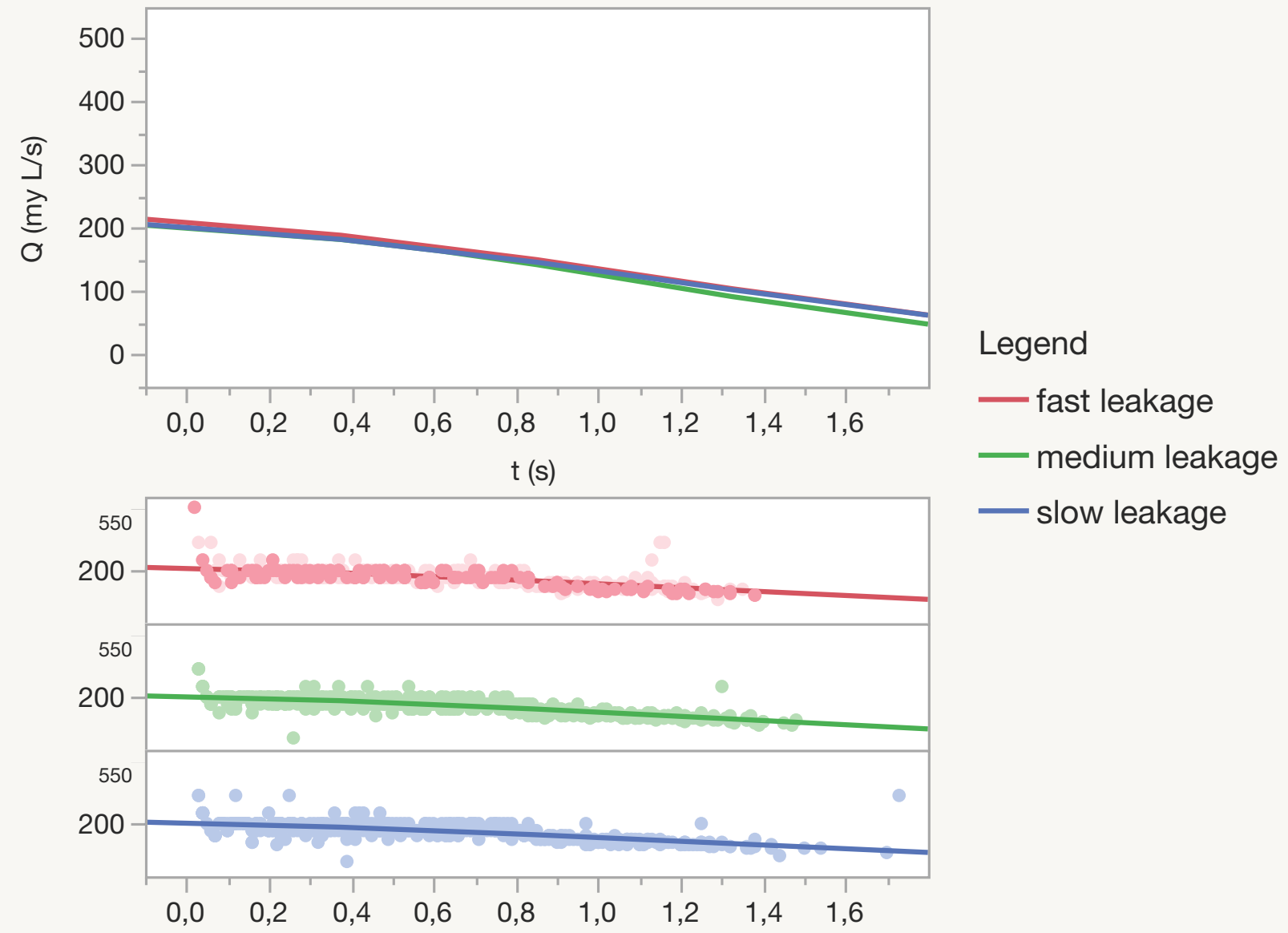

Figure 5: The flow rates when mimicking data for patient 2, 4, and 7 when G30 needle was used for injection into the in vitro model. Patient 2, 4, and 7 was chosen from the reference study because the same amount, $0.24 \mathrm{~mL}$, was injected into the patient during the in vivo study. 


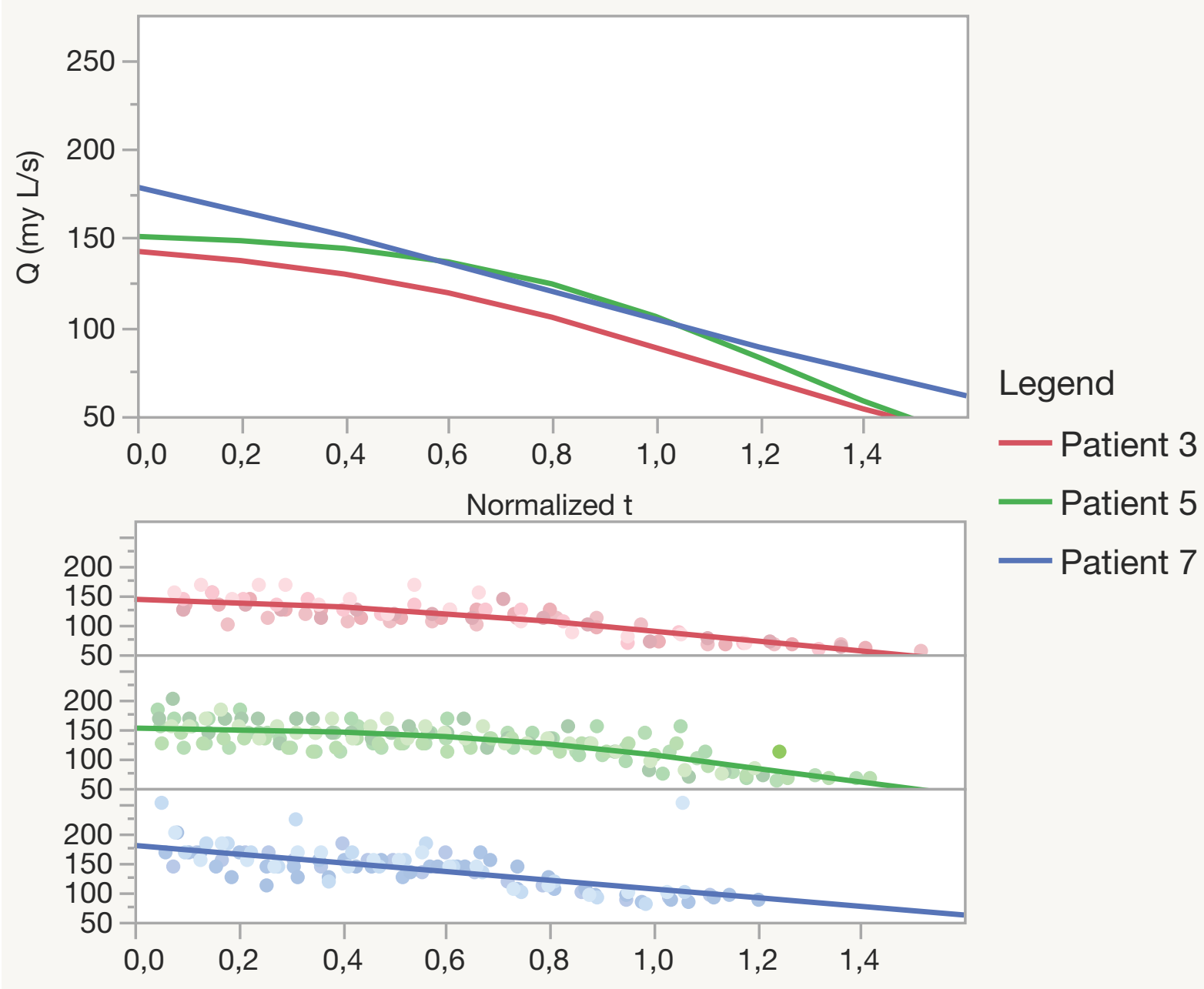

Figure 6: The data obtained when mimicking data for patient 3, 5, and 7 from the developed in vitro model when a G32 needle is used for injection. Patient 3, 5, and 7 was used from the reference study since they represent the top, middle, and the bottom of the interval seen in figure 1. 

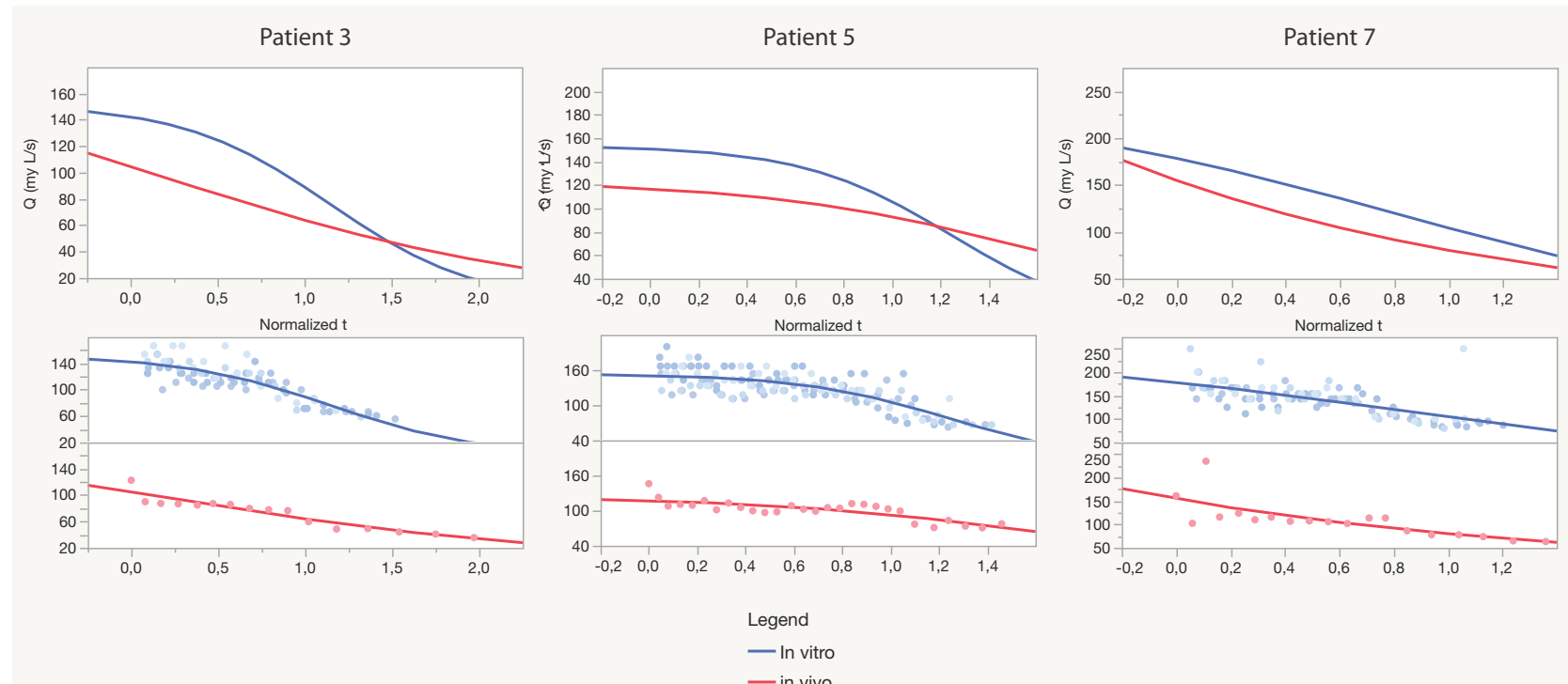

Figure 7: The comparison of patient 3, 5, and 7 in vivo data from the Thomsen et al. with the in vitro data obtained by the developed model when a G32 needle is used for injection. 